Homology, Homotopy and Applications, vol. 16(1), 2014, pp.27-47

\title{
SECONDARY MULTIPLICATION IN TATE COHOMOLOGY OF GENERALIZED QUATERNION GROUPS
}

\author{
MARTIN LANGER \\ (communicated by J. P. C. Greenlees)
}

\begin{abstract}
Let $k$ be a field, and let $G$ be a finite group. By a theorem of D. Benson, H. Krause, and S. Schwede, there is a canonical element in the Hochschild cohomology of the Tate cohomology $\gamma_{G} \in H H^{3,-1} \hat{H}^{*}(G)$ with the following property: Given any graded $\hat{H}^{*}(G)$-module $X$, the image of $\gamma_{G}$ in $\operatorname{Ext}_{\hat{H}^{*}(G)}^{3,-1}(X, X)$ is zero if and only if $X$ is isomorphic to a direct summand of $\hat{H}^{*}(G, M)$ for some $k G$-module $M$. In particular, if $\gamma_{G}=0$ then every module is a direct summand of a realizable $\hat{H}^{*}(G)$ module.

We prove that the converse of that last statement is not true by studying in detail the case of generalized quaternion groups. Suppose that $k$ is a field of characteristic 2 and $G$ is generalized quaternion of order $2^{n}$ with $n \geqslant 3$. We show that $\gamma_{G}$ is nontrivial for all $n$, but there is an $\hat{H}^{*}(G)$-module detecting this non-triviality if and only if $n=3$.
\end{abstract}

\section{Introduction}

Let $k$ be a field, $G$ a finite group, and let $\hat{H}^{*}(G)$ denote the graded Tate cohomology algebra of $G$ over $k$. The starting point of this paper is the following theorem of D. Benson, H. Krause, and S. Schwede:

Theorem 1.1. [2] There exists a canonical element in Hochschild cohomology of $\hat{H}^{*}(G)$

$$
\gamma_{G} \in H H^{3,-1} \hat{H}^{*}(G),
$$

such that for any graded $\hat{H}^{*}(G)$-module $X$, the following are equivalent:

(i) The image of $\gamma_{G}$ in $\operatorname{Ext}_{\hat{H}^{*}(G)}^{3,-1}(X, X)$ is zero.

(ii) There exists a $k G$-module $M$ such that $X$ is a direct summand of the graded $\hat{H}^{*}(G)$-module $\hat{H}^{*}(G, M)$.

Received November 23, 2009, revised January 26, 2010; published on February 18, 2014. 2010 Mathematics Subject Classification: 20J06, 55S35.

Key words and phrases: Tate cohomology, higher multiplication.

Article available at http://dx.doi.org/10.4310/HHA.2014.v16.n1.a2

Copyright (C) 2014, International Press. Permission to copy for private use granted. 
Let us call an $\hat{H}^{*}(G)$-module realizable if it is isomorphic to a module of the form $\hat{H}^{*}(G, M)$ for some $k G$-module $M$. As an immediate consequence we get the following.

Corollary 1.2. If $\gamma_{G}=0$, then every $\hat{H}^{*}(G)$-module is a direct summand of a realizable module.

At this point it is natural to ask for the converse of that statement. That is, given the fact that $\gamma_{G} \neq 0$, is there some $\hat{H}^{*}(G)$-module detecting the non-triviality of $\gamma_{G}$ ? Theorem 1.1 works more generally in the situation of differential graded algebras, and in that setup the converse of the corresponding corollary is known to the false: Benson, Krause, and Schwede provide an example of a dg algebra $A$ such that the canonical class $\gamma_{A} \in H H^{3,-1}\left(H^{*} A\right)$ is non-trivial, but every $H^{*} A$-module is realizable (see [2, Proposition 5.16]). Nevertheless, the author believes that the question whether there is such an example coming from Tate cohomology of groups is still open.

In this paper we will compute $\gamma_{G}$ explicitly for the generalized quaternion groups $G$. In what follows, let $t \geqslant 2$ be a power of 2 , and let $G=Q_{4 t}$ be the group of generalized quaternions

$$
Q_{4 t}=\left\langle g, h \mid g^{t}=h^{2}, g h g=h\right\rangle .
$$

Let $k$ be a field of characteristic 2 , and denote by $L=k G$ the group algebra of $G$ over $k$. Then the Tate cohomology ring $\hat{H}^{*}(G)$ is well known; it is given by

$$
\hat{H}^{*}\left(Q_{4 t}\right)=\widehat{\operatorname{Ext}}_{L}^{*}(k, k) \cong \begin{cases}k\left[x, y, s^{ \pm 1}\right] /\left(x^{2}+y^{2}=x y, y^{3}=0\right) & \text { if } t=2, \\ k\left[x, y, s^{ \pm 1}\right] /\left(x^{2}=x y, y^{3}=0\right) & \text { if } t \geqslant 4,\end{cases}
$$

with degrees $|x|=|y|=1,|s|=4$ (see, e.g., [4, Chapter XII $\S 11]$ and [1, IV Lemma $2.10]$ ). Our main goal is to prove the following theorem.

Theorem 1.3. The element $\gamma_{Q_{8}} \in H H^{3,-1} \hat{H}^{*}\left(Q_{8}\right)$ is non-trivial, and the cokernel of the map

$$
\hat{H}^{*}\left(Q_{8}\right)[-1] \oplus \hat{H}^{*}\left(Q_{8}\right)[-1] \stackrel{\left(\begin{array}{cc}
y & x+y \\
x & y
\end{array}\right)}{\longrightarrow} \hat{H}^{*}\left(Q_{8}\right) \oplus \hat{H}^{*}\left(Q_{8}\right)
$$

is a graded $\hat{H}^{*}\left(Q_{8}\right)$-module which is not a direct summand of a realizable one. For $t \geqslant 4$ the element $\gamma_{Q_{4 t}} \in H H^{3,-1} \hat{H}^{*}\left(Q_{4 t}\right)$ is non-trivial, but every graded $\hat{H}^{*}\left(Q_{4 t}\right)$ module is a direct summand of a realizable one.

The plan is as follows: In the first section we will briefly recall the definitions needed in Theorem 1.1; most of this part is taken from [2], and the reader interested in details should consult that source. In the second section we turn to the computation of a Hochschild cocycle $m$ representing the canonical class $\gamma_{G}$. In the third section we prove the statements about realizability of modules. Theorem 1.3 will then follow from Theorems 3.6, 3.8, 4.3, and Propositions 4.7 and 4.8.

\section{Acknowledgments}

Part of this paper is part of a Diploma thesis written at the Mathematical Institute, University of Bonn. I would like to thank my advisor, Stefan Schwede, for suggesting the subject and all his helpful comments on the project. I would also like to thank the referee of an earlier version of this paper for some helpful remarks. 


\section{Prerequisites}

\subsection{Notation and conventions}

All occurring modules will be right modules. We shall often work over a fixed ground field $k$; then $\otimes$ means tensor product over $k$. Whenever convenient, we write $\left(a_{1}, a_{2}, \ldots, a_{n}\right)$ instead of $a_{1} \otimes a_{2} \otimes \cdots \otimes a_{n}$. If $G$ is a group, then $k$ is often considered as a trivial $k G$-module.

Let $R$ be a ring with unit, and let $M$ be a $\mathbb{Z}$-graded $R$-module. The degree of every (homogeneous) element $m \in M$ will be denoted by $|m|$. For every integer $n$ the module $M[n]$ is defined by $M[n]^{j}=M^{n+j}$ for all $j$. Given two such modules $M$ and $L$, a morphism $f: L \longrightarrow M$ is a family $f^{j}: L^{j} \longrightarrow M^{j}$ of $R$-module homomorphisms. The group of all these morphisms is denoted by $\operatorname{Hom}_{R}(L, M)$. Furthermore, we have $\operatorname{Hom}_{R}^{m}(L, M)=\operatorname{Hom}_{R}(L, M[m])$, the morphisms of degree $m$. The graded module $L \otimes M$ is given by $(L \otimes M)^{m}=\bigoplus_{i+j=m} L^{i} \otimes M^{j}$. If $M$ is a differential graded $R$ module with differential $d$, then the differential of $M[n]$ is given by $(-1)^{n} d$.

\subsection{Tate Cohomology}

Let us recall briefly the definition and basic properties of Tate cohomology. Let $k$ be a field, and let $G$ be a finite group. Then $L=k G$ is a self-injective algebra (i.e., the classes of projective and injective right- $L$-modules coincide). For any $L$-module $N$ we get a complete projective resolution $P_{*}$ of $N$ by splicing together a projective and an injective resolution of $N$ :

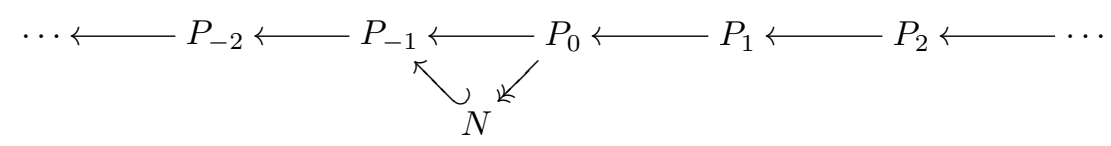

Given another $L$-module $M$, we can apply the functor $\operatorname{Hom}_{L}(-, M)$ to $P_{*}$; then Tate cohomology is defined to be the cohomology groups of the resulting complex:

$$
\widehat{\operatorname{Ext}}_{L}^{n}(N, M)=H^{n}\left(\operatorname{Hom}_{L}\left(P_{*}, M\right)\right) \text { for all } n \in \mathbb{Z} \text {. }
$$

For arbitrary $L$-modules $X, Y$, and $Z$, we have a cup product

$$
\widehat{\operatorname{Ext}}_{L}^{m}(Y, Z) \otimes \widehat{\operatorname{Ext}}_{L}^{n}(X, Y) \longrightarrow \widehat{\operatorname{Ext}}_{L}^{m+n}(X, Z) ;
$$

see, e.g., $[3, \S 6]$. Therefore, $\hat{H}^{*}(G)=\hat{H}^{*}(G, k)=\widehat{\operatorname{Ext}}_{k G}^{*}(k, k)$ is a graded algebra, and $\hat{H}^{*}(G, M)=\widehat{\operatorname{Ext}}_{k G}^{*}(k, M)$ is a graded $\hat{H}^{*}(G)$-module for every $k G$-module $M$. We call a graded $\hat{H}^{*}(G)$-module $X$ realizable if it is isomorphic to $\hat{H}^{*}(G, M)$ for some $k G$-module $M$.

There is another way of describing the product of $\hat{H}^{*}(G)$, in terms of $P_{*}$. Consider the differential graded algebra $\mathcal{A}=\operatorname{Hom}_{L}^{*}\left(P_{*}, P_{*}\right.$ ), which (in degree $n$ ) is given by

$$
\mathcal{A}^{n}=\prod_{j \in \mathbb{Z}} \operatorname{Hom}_{L}\left(P_{j+n}, P_{j}\right)
$$

and the differential $d: \mathcal{A}^{n} \longrightarrow \mathcal{A}^{n+1}$ is defined to be

$$
(d f)_{j}=\partial \circ f_{j+1}-(-1)^{n} f_{j} \circ \partial .
$$

Here $\partial$ denotes the differential of $P_{*}$. $\mathcal{A}$ is called the endomorphism dga of $P$. With this definition, the cocycles of $\mathcal{A}$ (of degree $n$ ) are exactly the chain transformations 
$P[n] \rightarrow P$, and two cocycles differ by a coboundary if and only if they are chain homotopic. Using standard arguments from homological algebra, one shows that the following map is an isomorphism of $k$-vector spaces:

$$
\begin{aligned}
H^{n} \mathcal{A} & \cong \widehat{\operatorname{Ext}}_{L}^{n}(k, k), \\
{[f] } & \mapsto\left[\epsilon \circ f_{0}\right] .
\end{aligned}
$$

Here $\epsilon: P_{0} \longrightarrow k$ is the augmentation. This isomorphism is compatible with the multiplicative structures. We will often write $\bar{a}$ for elements of the endomorphism dga; if $\bar{a}$ is a cocycle, then $a$ denotes the corresponding cohomology class.

\subsection{Hochschild Cohomology}

We now give a short review of Hochschild cohomology. Let $\Lambda$ be a graded algebra over the field $k$, and let $M$ be a graded $\Lambda$ - $\Lambda$-bimodule, the elements of $k$ acting symmetrically. Define a cochain complex $C^{\bullet, *}(\Lambda, M)$ by

$$
C^{n, m}(\Lambda, M)=\operatorname{Hom}_{k}^{m}\left(\Lambda^{\otimes n}, M\right),
$$

with a differential $\delta$ of bidegree $(1,0)$ given by

$$
\begin{aligned}
& (\delta \varphi)\left(\lambda_{1}, \ldots, \lambda_{n+1}\right)=(-1)^{m\left|\lambda_{1}\right|} \lambda_{1} \varphi\left(\lambda_{2}, \ldots, \lambda_{n+1}\right) \\
& \quad+\sum_{i=1}^{n}(-1)^{i} \varphi\left(\lambda_{1}, \ldots, \lambda_{i} \lambda_{i+1}, \ldots, \lambda_{n+1}\right)+(-1)^{n+1} \varphi\left(\lambda_{1}, \ldots, \lambda_{n}\right) \lambda_{n+1} .
\end{aligned}
$$

The Hochschild cohomology groups $H H^{*, *}(\Lambda, M)$ are defined as the cohomology groups of that complex:

$$
H H^{s, t}(\Lambda, M)=H^{s}\left(C^{*, t}(\Lambda, M)\right)
$$

In particular, we can regard $M=\Lambda$ as a bimodule over itself; we will then write $H H^{s, t}(\Lambda)=H H^{s, t}(\Lambda, \Lambda)$. For example, an element of $H H^{3,-1}(\Lambda)$ is represented by a family of $k$-linear maps

$$
m=\left\{m_{i, j, l}: \Lambda^{i} \otimes \Lambda^{j} \otimes \Lambda^{l} \longrightarrow \Lambda^{i+j+l-1}\right\}_{i, j, l \in \mathbb{Z}}
$$

satisfying the cocycle relation

$$
(-1)^{|a|} a \cdot m(b, c, d)-m(a b, c, d)+m(a, b c, d)-m(a, b, c d)+m(a, b, c) \cdot d=0
$$

for all $a, b, c, d \in \Lambda$.

Whenever $X$ and $Y$ are $\Lambda$ - $\Lambda$-bimodules, one has a cup product pairing

$$
\cup: \operatorname{Hom}_{\Lambda}(X, Y) \otimes H H^{*, *} \Lambda \longrightarrow \operatorname{Ext}_{\Lambda}^{*, *}(X, Y) \text {. }
$$

Here $\operatorname{Ext}_{\Lambda}^{s, t}(X, Y)$ is defined to be $\operatorname{Ext}_{\Lambda}^{s}(X, Y[t])$. In particular, we have the map

$$
\begin{aligned}
H H^{3,-1} \hat{H}^{*}(G) & \longrightarrow \operatorname{Ext}_{\hat{H}^{*}(G)}^{3,-1}(X, X) \\
\phi & \mapsto \operatorname{id}_{X} \cup \phi
\end{aligned}
$$

for every $\hat{H}^{*}(G)$-module $X$. This is the map occurring in Theorem 1.1.

\subsection{The canonical element $\gamma$}

We are now going to describe the construction of the element $\gamma$ mentioned in Theorem 1.1. More generally, we will construct an element $\gamma_{\mathcal{A}} \in H H^{3,-1} H^{*} \mathcal{A}$ for 
every differential graded algebra $\mathcal{A}$ over $k$; then we can take $\mathcal{A}$ to be the endomorphism algebra of a complete projective resolution of $k$ as a trivial $k G$-module to get $\gamma_{G} \in$ $H H^{3,-1} \hat{H}^{*}(G)$.

For a dg-algebra $\mathcal{A}$, consider $H^{*} \mathcal{A}$ as a differential graded $k$-module with trivial differential. Then choose a morphism of dg- $k$-modules $f_{1}: H^{*} \mathcal{A} \longrightarrow \mathcal{A}$ of degree 0 which induces the identity in cohomology. This is the same as choosing a representative in $\mathcal{A}$ for every class in $H^{*} \mathcal{A}$ in a $k$-linear way. For every two elements $x, y \in H^{*} \mathcal{A}$, $f_{1}(x y)-f_{1}(x) f_{1}(y)$ is null-homotopic; therefore, we can choose a morphism of graded modules

$$
f_{2}: H^{*} \mathcal{A} \otimes H^{*} \mathcal{A} \longrightarrow \mathcal{A}
$$

of degree -1 such that for all $x, y \in H^{*} \mathcal{A}$, we have

$$
d f_{2}(x, y)=f_{1}(x y)-f_{1}(x) f_{1}(y) .
$$

Then for all $a, b, c \in H^{*} \mathcal{A}$,

$$
f_{2}(a, b) f_{1}(c)-f_{2}(a, b c)+f_{2}(a b, c)-(-1)^{|a|} f_{1}(a) f_{2}(b, c)
$$

is a cocycle in $\mathcal{A}$, the cohomology class of which will be denoted by $m(a, b, c)$. This defines a map $m:\left(H^{*} \mathcal{A}\right)^{\otimes 3} \longrightarrow H^{*} \mathcal{A}$ of degree -1 . An explicit computation shows that $m$ is a Hochschild cocycle, thereby representing a class $\gamma_{A} \in H H^{3,-1} H^{*} \mathcal{A}$. This class is independent of the choices made.

\section{Computation of the canonical element}

From now on, let $k$ be a field of characteristic 2 . Let $t \geqslant 2$ be a power of 2 , and let $G=Q_{4 t}$ be the group of generalized quaternions

$$
Q_{4 t}=\left\langle g, h \mid g^{t}=h^{2}, g h g=h\right\rangle .
$$

We denote by $k G$ the group algebra of $G$ over $k$, and $F=k G$ denotes the free module of rank 1 over that algebra. In this section, we are going to explicitly compute a Hochschild cochain $m$ representing the canonical class $\gamma_{G}$.

\subsection{The class of a map}

We begin with an observation that will reduce the subsequent computations somewhat. Let us recall the construction of a representative of $\gamma_{G}$. First of all, we have to construct a projective resolution $P$, and we will actually find a minimal projective resolution. Then we have to choose a cycle selection homomorphism $f_{1}: \hat{H}^{*}(G) \rightarrow$ $\operatorname{Hom}_{k G}^{*}(P, P)$ such that any class $a$ is mapped to a representative $f_{1}(a)$. We can find a $k$-linear map $f_{2}: \hat{H}^{*}(G) \otimes \hat{H}^{*}(G) \rightarrow \operatorname{Hom}_{k G}^{*}(P, P)$ of degree -1 satisfying $d f_{2}(a, b)=f_{1}(a) f_{1}(b)-f_{1}(a b)$ for all $a, b$. Finally, we are interested in terms of the form

$$
f_{2}(a, b) f_{1}(c)+f_{2}(a, b c)+f_{2}(a b, c)+f_{1}(a) f_{2}(b, c) ;
$$

this is a cocycle in $\operatorname{Hom}_{k G}^{*}(P, P)$. In order to determine the class of this cocycle, it is enough to know the degree 0 map of it (cf. (1)). This observation leads to the following definition. 
Definition 3.1. For every $f \in \operatorname{Hom}_{k G}^{n}(P, P)$, i.e., a family of maps $f_{j}: P_{j+n} \rightarrow P_{j}$ $(j \in \mathbb{Z})$, not necessarily commuting with the differential, we denote by $\mathcal{C}(f)$ the class of the map $\epsilon \circ f_{0}: P_{n} \rightarrow k$ in $H^{n} \operatorname{Hom}_{k G}\left(P_{*}, k\right)=\hat{H}^{n}(G)$.

Note that the complex $\operatorname{Hom}_{k G}\left(P_{*}, k\right)$ has trivial differential; thus, every element in $\operatorname{Hom}_{k G}\left(P_{*}, k\right)$ and in particular $\epsilon \circ f_{0}$ is a cocycle. The definition above gives a map

$$
\begin{aligned}
\mathcal{C}: \operatorname{Hom}_{k G}^{n}(P, P) & \longrightarrow \hat{H}^{n}(G) \\
f & \mapsto\left[\epsilon \circ f_{0}\right] .
\end{aligned}
$$

Proposition 3.2. The map $\mathcal{C}$ has the following properties:

(i) If $f \in \operatorname{Hom}_{k G}^{n}(P, P)$ is a cocycle, then $\mathcal{C}(f)$ is the cohomology class of $f$; in particular, $\mathcal{C} \circ f_{1}=$ id.

(ii) The map $\mathcal{C}$ is k-linear.

(iii) If $\mathcal{C}\left(f_{1}\right)=\mathcal{C}\left(f_{2}\right)$ for some $f_{1}, f_{2} \in \operatorname{Hom}_{k G}^{n}(P, P)$, then $\mathcal{C}\left(f_{1} g\right)=\mathcal{C}\left(f_{2} g\right)$ for all $g \in \operatorname{Hom}_{k G}^{m}(P, P)$.

(iv) If $a \in \operatorname{Hom}_{k G}^{m}(P, P)$ is a cocycle and $f \in \operatorname{Hom}_{k G}^{n}(P, P)$ is an arbitrary element, then $\mathcal{C}(f a)=\mathcal{C}(f) \mathcal{C}(a)$.

Proof. (i) follows from (1). (ii) holds by definition. (iii): If $\mathcal{C}\left(f_{i}\right)=0$, then $\epsilon \circ f_{i}=0$. This implies $\epsilon \circ f_{i} \circ g=0$; hence $\mathcal{C}\left(f_{i} g\right)=0$. For general $f_{1}, f_{2}$, note $\mathcal{C}\left(f_{1}-f_{2}\right)=0$; by what we just proved, $\mathcal{C}\left(\left(f_{1}-f_{2}\right) g\right)=0$ and therefore $\mathcal{C}\left(f_{1} g\right)=\mathcal{C}\left(f_{2} g\right)$. (iv): Choose a cocycle $h \in \operatorname{Hom}_{k G}^{n}(P, P)$ satisfying $\mathcal{C}(h)=\mathcal{C}(f)$. Then by (iii)

$$
\mathcal{C}(f a)=\mathcal{C}(h a)=\mathcal{C}(h) \mathcal{C}(a)=\mathcal{C}(f) \mathcal{C}(a) .
$$

The following corollary will simplify computations later on.

Proposition 3.3. The map $f_{2}$ can be chosen in such a way that $\mathcal{C} \circ f_{2}=0$.

Proof. Choose any $\tilde{f}_{2}$ (satisfying $\left.d \tilde{f}_{2}(a, b)=f_{1}(a) f_{1}(b)-f_{1}(a b)\right)$. Put $f_{2}=\tilde{f}_{2}-f_{1} \circ$ $\mathcal{C} \circ \tilde{f}_{2}$. Since $d f_{1}=0$, we get

$$
d f_{2}(a, b)=d \tilde{f}_{2}(a, b)=f_{1}(a) f_{1}(b)-f_{1}(a b),
$$

and from $\mathcal{C} \circ f_{1}=$ id, it follows that

$$
\mathcal{C} \circ f_{2}=\mathcal{C} \circ \tilde{f}_{2}-\mathcal{C} \circ f_{1} \circ \mathcal{C} \circ \tilde{f}_{2}=0 .
$$

Consider (2) with this simplified version of $f_{2}$. By applying $\mathcal{C}$, we get the term

$$
\mathcal{C}\left(f_{2}(a, b) f_{1}(c)\right)+\mathcal{C}\left(f_{2}(a, b c)\right)+\mathcal{C}\left(f_{2}(a b, c)\right)+\mathcal{C}\left(f_{1}(a) f_{2}(b, c)\right) .
$$

This is the cohomology class of (2). Note that the individual terms $f_{2}(a, b) f_{1}(c)$, $f_{2}(a, b c) \ldots$ will not be cocycles in general, but the map $\mathcal{C}$ assigns cohomology classes to them in such a way that the sum will be the class we are looking for.

By our choice of $f_{2}$ (such that $\mathcal{C} \circ f_{2}=0$ ), the first three terms in the sum vanish (note that $\mathcal{C}\left(f_{2}(a, b) f_{1}(c)\right)=\mathcal{C}\left(f_{2}(a, b)\right) c$ by Proposition 3.2.(iv)). Thus we are interested in terms of the form $\mathcal{C}\left(f_{1}(a) f_{2}(b, c)\right)$, where $a, b, c$ run through all elements of a $k$-basis of $\hat{H}^{*}(G)$. 


\subsection{Generating cocycles and homotopies}

Now we start the actual computation of $\gamma$. We begin with the construction of a minimal projective resolution $P$ and some cocycles in the endomorphism dga of $P$. Let us define some elements of the group algebra $k G$ as follows. Put $a=g+1$, $b=h+1$ and $c=h g+1$. Furthermore, we write $N=\sum_{j \in G} j$ for the norm element. Here are some formulae we will frequently use:

$$
\begin{aligned}
a^{t} & =b^{2}=c^{2}, & a^{2 t} & =b^{4}=0, \\
b a & =a c=a+b+c, & N & =a^{2 t-1} b, \\
c & =a+b g, & g c & =a+b, \\
N & =c a^{2 t-2} b=c a^{2 t-1}, & N & =a^{2 t-1}+a^{2 t-2} b+c a^{2 t-2}, \\
c a^{t-1} b & =c a^{t-1}+a^{t-1} b . & &
\end{aligned}
$$

Also note that $a^{2 t-1}, a^{2 t-2}$, and $a^{2 t-4}$ lie in the center of $k Q_{4 t}$. Now a 4-periodic complete projective resolution of the trivial $k G$-module $k$ is given as follows (see $[4$, Chapter XII $\S 7])$ :

$$
\cdots \stackrel{N}{\longleftarrow} P_{0}=F \stackrel{(a b)}{\longleftarrow} P_{1}=F^{2} \stackrel{\left(\begin{array}{ccc}
a^{t-1} & c \\
b & a
\end{array}\right)}{\longleftarrow} P_{2}=F^{2} \stackrel{\left(\begin{array}{c}
a \\
c
\end{array}\right)}{\longleftarrow} P_{3}=F \stackrel{N}{\longleftarrow} P_{4}=F \longleftarrow \cdots
$$

Since the resolution is minimal, the differential of the complex $\operatorname{Hom}_{k G}\left(P_{*}, k\right)$ vanishes; therefore, we immediately get the well-known additive structure of $\hat{H}^{*}(G)$ :

$$
\hat{H}^{4 n}(G) \cong \hat{H}^{4 n+3}(G) \cong k, \quad \hat{H}^{4 n+1}(G) \cong \hat{H}^{4 n+2}(G) \cong k^{2} .
$$

Let us write $\bar{s}: P \rightarrow P[4]$ for the shift map, given by the identity map in every degree. This is an invertible cocycle; thus, multiplication by a suitable power of $s$ yields an isomorphism $\hat{H}^{4 n+u}(G) \cong \hat{H}^{u}(G)$ for $u=0,1,2,3$ and $n \in \mathbb{Z}$. Now we are heading for explicit generators $x, y$ of $\hat{H}^{1}(G) \cong H^{1} \operatorname{Hom}_{k G}^{*}(P, P)$, which are represented by chain maps $\bar{x}, \bar{y}: P[1] \rightarrow P$. By construction, we have $P_{1}=F^{2}$ and $P_{0}=F$. We extend the two projections $P_{1} \rightarrow P_{0}$ to chain transformations $P[1] \rightarrow P$ as follows: For $\bar{x}: P \rightarrow$ $P[1]$ we take

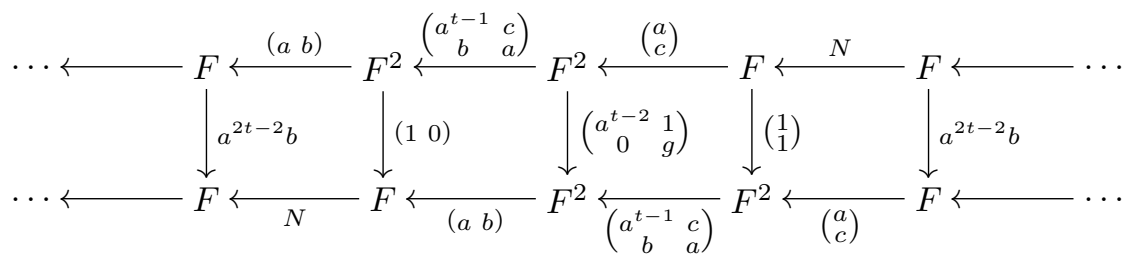

and extend this 4-periodically. The 4-periodic chain map $\bar{y}: P \rightarrow P[1]$ is defined as follows:

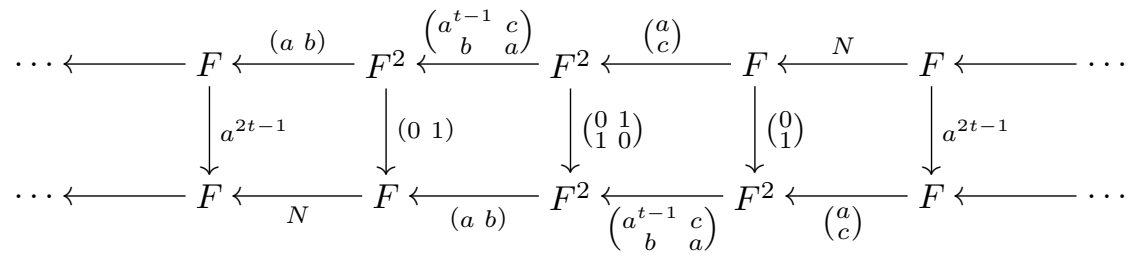


Since these cocycles are 4-periodic, they commute with $\bar{s}$. Let us determine the pairwise products of these maps. We start with $\bar{x} \bar{y}$ :

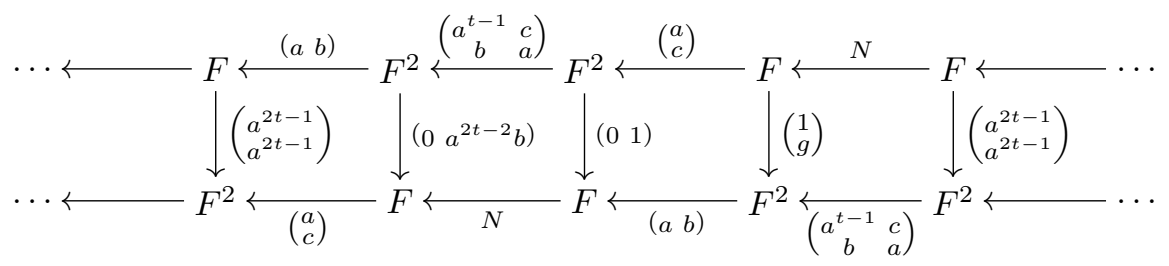

The product $\bar{y} \bar{x}$ is given as follows:

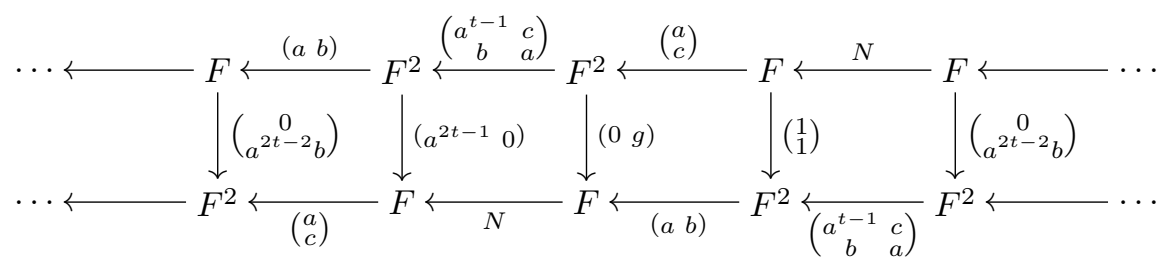

Next, we compute $\bar{x}^{2}$ :

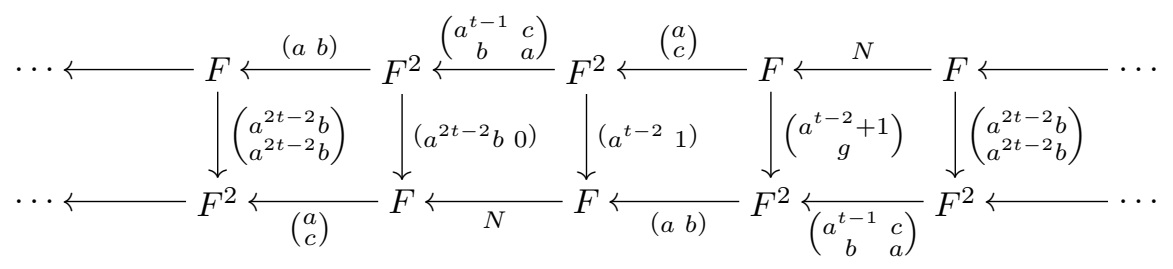

And now $\bar{y}^{2}$ :

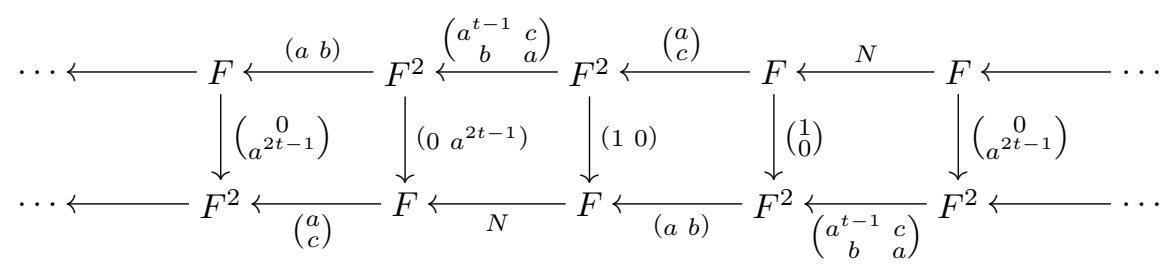

In each of these cocycles, the map $P_{2} \rightarrow P_{0}$ determines the cohomology class by the isomorphism (1); in $k^{2}$, they correspond to $(01),(01),\left(\epsilon\left(a^{t-2}\right) 1\right)$, and (10), respectively. Hence, $\hat{H}^{2}(G)$ is generated by $x^{2}$ and $y^{2}$, and we have $x y=y x$. Furthermore, we also see from this description that

$$
x y= \begin{cases}x^{2}+y^{2} & \text { if } t=2, \\ x^{2} & \text { otherwise }\end{cases}
$$

But we will need explicit chain homotopies for all these relations later on, so let us start with the commutator relation $x y=y x$. Let $\bar{p}$ be the 4-periodic null-homotopy 
for $\bar{x} \bar{y}+\bar{y} \bar{x}$ defined as follows:

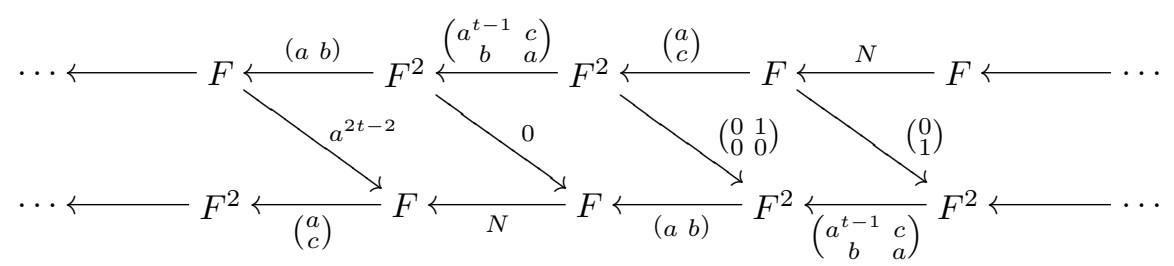

Now let us compute $\bar{y}^{3}$ :

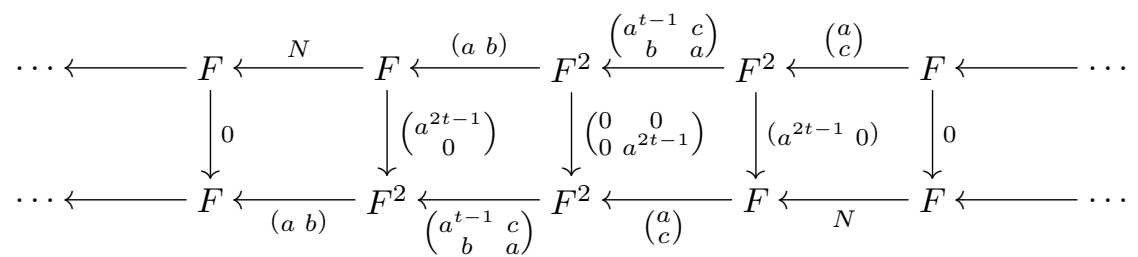

Then we find a null-homotopy for that map in two steps: First, consider the 4-periodic extension of the map

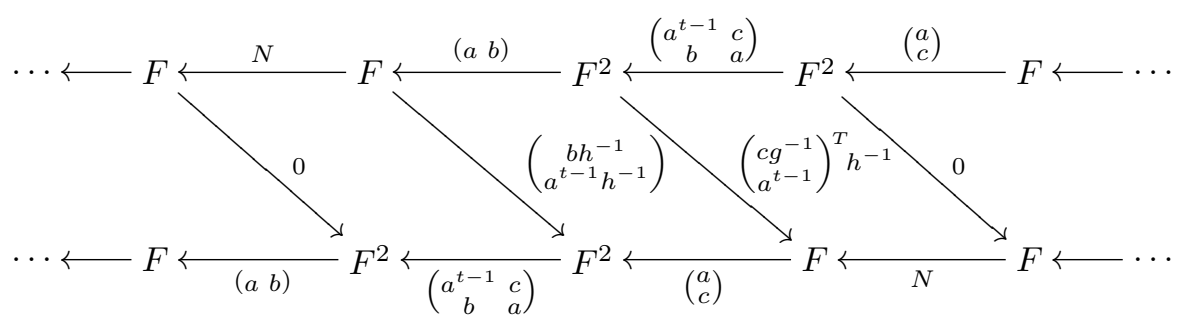

and call it $\bar{w}^{\prime}$. Note that this will not quite be a homotopy for $\bar{y}^{3}$, because it yields the wrong result in degrees $P_{4 n+2} \rightarrow P_{4 n-1}$ for all $n \in \mathbb{Z}$. But if we put

$$
P_{8 n+j+3} \rightarrow P_{8 n+j}: \bar{w}_{8 n+j}= \begin{cases}\bar{w}_{8 n+j}^{\prime} & \text { if } j=0,1,2,3, \\ \left(\bar{w}^{\prime}+\bar{y}^{2}\right)_{8 n+j} & \text { if } j=4,5,6,7,\end{cases}
$$

then we get an 8-periodic null-homotopy for $\bar{y}^{3}$ which will be called $\bar{w}$ and satisfies $\bar{s} \bar{w}+\bar{w} \bar{s}=\bar{y}^{2} \bar{s}$.

\subsection{Computation for the quaternion group}

Due to the different multiplicative relation in $\hat{H}^{*}(G)$, we need to consider the cases $t=2$ and $t \geqslant 4$ separately. We start with $t=2$. In this case, the map

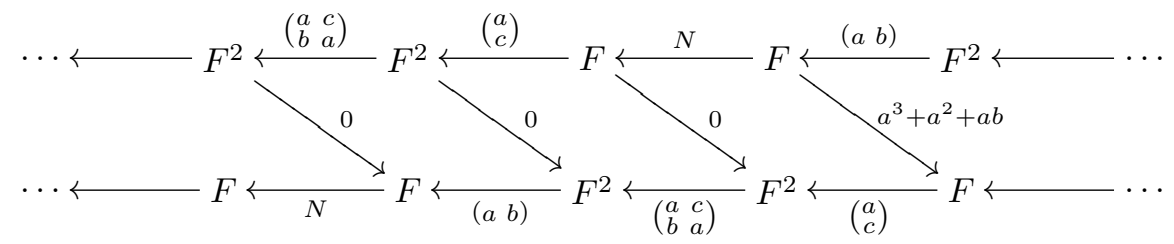

can be extended (as we did with $\bar{w}$ above) to an 8-periodic null-homotopy $\bar{r}$ for $\bar{x}^{2}+\bar{x} \bar{y}+\bar{y}^{2}$ satisfying $\bar{s} \bar{r}+\bar{r} \bar{s}=(\bar{x}+\bar{y}) \bar{s}$. Notice that $\bar{x} \bar{y}^{2}: P_{3} \rightarrow P_{0}$ is the identity 
map, which implies that $x y^{2} \neq 0 \in \hat{H}^{3}(G)$. Gathering the results we obtained so far, we recover the known fact that

$$
\hat{H}^{*}(G) \cong k\left[x, y, s^{ \pm 1}\right] /\left(x^{2}+y^{2}=x y, y^{3}=0\right) .
$$

Let us remark here that all monomials in $x$ and $y$ of degree bigger than 3 vanish in this ring.

Proposition 3.4. Let $\alpha, \beta, \gamma$ be monomials in the (non-commutative) variables $\bar{x}, \bar{y}$, and assume that the degree $|\beta| \geqslant 3$. Then we have the following formulae:

$$
\begin{aligned}
\mathcal{C}(\bar{p} \alpha) & =0, & \mathcal{C}(\bar{r} \alpha)=0, & \mathcal{C}(\bar{w} \alpha)=0, \\
\mathcal{C}(\bar{x} \bar{p} \alpha) & =x y \mathcal{C}(\alpha), & \mathcal{C}(\gamma \bar{r} \alpha)=0, & \mathcal{C}(\gamma \bar{w} \alpha)=0, \\
\mathcal{C}(\bar{y} \bar{p} \alpha) & =0, & & \\
\mathcal{C}\left(\bar{x}^{2} \bar{p} \alpha\right) & =x^{2} y \mathcal{C}(\alpha), & & \\
\mathcal{C}\left(\bar{y}^{2} \bar{p} \alpha\right) & =0, & & \\
\mathcal{C}(\beta \bar{p} \alpha) & =0 . & &
\end{aligned}
$$

Proof. By Proposition 3.2.(iii) we can assume that the degree of $\beta$ is at most 3 . Furthermore, we can assume $\alpha=1$ by Proposition 3.2.(iv). In order to determine $\mathcal{C}(\bar{a} \bar{w})$ for any given cocycle $\bar{a}$ of degree $n$, we consider the composition

$$
P_{n+2} \stackrel{\bar{w}_{n}}{\longrightarrow} P_{n} \stackrel{\bar{a}_{0}}{\rightarrow} P_{0} \stackrel{\epsilon}{\rightarrow} k
$$

as an element of $H^{n+2} \operatorname{Hom}_{k G}\left(P_{*}, k\right)$. Notice $\operatorname{im}\left(\bar{w}_{n}\right) \subset \operatorname{ker}(\epsilon) \cdot P_{n}$. Therefore, $\operatorname{im}\left(\bar{a}_{0} \circ\right.$ $\left.\bar{w}_{n}\right) \subset \operatorname{ker}(\epsilon) \cdot P_{0}=\operatorname{ker}(\epsilon)$, and hence $\epsilon \circ \bar{a}_{0} \circ \bar{w}_{n}=0$. The same proof works for $\bar{r}$ instead of $\bar{w}$, so we are left with $\bar{p}$. For $\mathcal{C}(\bar{x} \bar{p})$, consider $\bar{x} \bar{p}$ in degree 0; i.e.,

$$
P_{2} \underset{\left(\begin{array}{ll}
0 \\
0 & 0
\end{array}\right)}{\stackrel{\bar{p}_{1}}{\longrightarrow}} P_{1} \underset{\left(\begin{array}{ll}
1 & 0
\end{array}\right)}{\stackrel{\bar{x}_{0}}{\longrightarrow}} P_{0} .
$$

This equals ( 01$): P_{2} \longrightarrow P_{0}$, which corresponds to $x y$. The remaining cases can be shown analogously.

Remark 3.5. Using $\mathcal{C}$, we can prove that there is no 4-periodic null-homotopy for $\bar{x}^{2}+\bar{x} \bar{y}+\bar{y}^{2}$ as follows: Suppose there is a 4-periodic null-homotopy; call it $\hat{r}$. Since $d(\hat{r}-\bar{r})=0, \bar{q}=\hat{r}-\bar{r}$ is a cocycle, representing some class $q$. By construction, $\bar{s} \bar{r}=$ $(\bar{r}+\bar{x}+\bar{y}) \bar{s}$. Since $\hat{r}$ is 4-periodic, we have $\mathcal{C}(\bar{s} \bar{q})=\mathcal{C}(\bar{q} \bar{s})-\mathcal{C}((\bar{x}+\bar{y}) \bar{s})=q s-(x+$ $y) s$ by Proposition 3.2. On the other hand, $\mathcal{C}(\bar{s} \bar{q})=s q$, and hence $(x+y) s=0$, a contradiction. In a similar way, one shows that there is no 4-periodic null-homotopy for $\bar{x}^{3}$.

As a next step, we are going to define the functions $f_{1}$ and $f_{2}$. A $k$-basis of $\hat{H}^{*}(G)$ is given by $\mathfrak{C}=\left\{s^{i}, x s^{i}, y s^{i}, x^{2} s^{i}, y^{2} s^{i}, x^{2} y s^{i} \mid i \in \mathbb{Z}\right\}$. Define the $k$-linear map $f_{1}$ on the basis $\mathfrak{C}$ by

$$
\begin{aligned}
f_{1}: \hat{H}^{*}(G) & \rightarrow \operatorname{Hom}_{k G}^{*}(P, P) \\
x^{\varepsilon} y^{\delta} s^{i} & \mapsto \bar{x}^{\varepsilon} \bar{y}^{\delta} \bar{s}^{i}
\end{aligned}
$$

for all $i, \varepsilon, \delta \in \mathbb{Z}$ for which the expression on the left-hand side lies in $\mathfrak{C}$. Let us define the set $\mathcal{B}=\left\{1, x, y, x^{2}, y^{2}, x^{2} y\right\}$. For all $b, c \in \mathcal{B}$ and $i, j \in \mathbb{Z}$, we have $f_{1}\left(b s^{i} c s^{j}\right)=$ 
$f_{1}(b c) \bar{s}^{i+j}$ and $f_{1}\left(b s^{i}\right) f_{1}\left(c s^{i}\right)=f_{1}(b) f_{1}(c) \bar{s}^{i+j}$, since $\bar{s}$ commutes with both $\bar{x}$ and $\bar{y}$. This implies that we can define $f_{2}$ on $\mathcal{B} \times \mathcal{B}$ and then extend it to $\mathfrak{C} \times \mathfrak{C}$ via $f_{2}\left(b s^{i}, c s^{j}\right)=f_{2}(b, c) \bar{s}^{i+j}$. Now define $f_{2}$ on $\mathcal{B} \times \mathcal{B}$ as follows:

\begin{tabular}{c|ccc|}
$f_{2}(b, c)$ & \multicolumn{4}{c}{$c$} \\
\\
\hline 1 & 0 & $x$ & 0 \\
\hline$x$ & 0 & 0 & $\bar{r}$ \\
$b$ & 0 & $\bar{p}+\bar{r}$ & 0 \\
$b$ & 0 & $\bar{x} \bar{r}+\bar{r} \bar{y}+\bar{w}$ & 0 \\
$x^{2}$ & 0 & $\bar{y} \bar{p}+\bar{y} \bar{r}+\bar{w}+\bar{p} \bar{x}+\bar{x} \bar{p}+\bar{x} \bar{y}$ & $\bar{w}$ \\
$y^{2}$ & 0 & $\bar{x} \bar{y}^{2}+\bar{x} \bar{w}+\bar{y} \bar{w}$ \\
$x^{2} y$ & 0 & $\bar{x}^{2} \bar{p}+\bar{x} \bar{r} \bar{y}+\bar{r} \bar{y}^{2}+\bar{w} \bar{y}+\bar{x}^{2} \bar{y}$ & \\
\hline
\end{tabular}

\begin{tabular}{c|ccc|} 
& $x^{2}$ & $y^{2}$ & $x^{2} y$ \\
\hline 1 & 0 & 0 & 0 \\
$x$ & $\bar{x} \bar{r}+\bar{r} y+\bar{w}$ & $\bar{r} \bar{y}+\bar{w}$ & $\bar{x} \bar{r} \bar{y}+\bar{r} \bar{y}^{2}+\bar{w} \bar{y}$ \\
$b$ & $\bar{p} \bar{x}+\bar{x} \bar{p}+\bar{x} \bar{y}$ & $\bar{w}$ & $\bar{y} \bar{r} \bar{y}+\bar{p} \bar{y}^{2}+\bar{x} \bar{w}+\bar{y} \bar{w}$ \\
$x^{2}$ & $\bar{x} \bar{r} \bar{x}+\bar{r} \bar{y} \bar{x}+\bar{w} \bar{x}$ & $\bar{r} \bar{y}^{2}+\bar{x} \bar{w}+\bar{y} \bar{w}$ & $*$ \\
$y^{2}$ & $\bar{y}^{2} \bar{r}+\bar{y}^{2} \bar{p}+\bar{w} \bar{x}+\bar{w} \bar{y}$ & $\bar{w} \bar{y}$ & $*$ \\
$x^{2} y$ & $*$ & $*$ & $*$ \\
\hline
\end{tabular}

Direct verification shows that $d f_{2}(b, c)=f_{1}(b c)-f_{1}(b) f_{1}(c)$ for all $b, c$ for which $f_{2}$ is defined. Each $*$ can be replaced by a suitable polynomial expression in $\bar{x}, \bar{y}, \bar{p}, \bar{r}, \bar{w}$ such that $d f_{2}(b, c)=f_{1}(b c)-f_{1}(b) f_{1}(c)$ holds for all $b, c$; as we will see, it does not matter which choice we make here. Our $f_{2}$ will then already be simplified in the sense of Proposition 3.3, which is why some apparently unnecessary terms occur (e.g., the $\bar{x} \bar{y}$ in $f_{2}\left(y, x^{2}\right)$ ). Indeed, $\mathcal{C} \circ f_{2}=0$, as one can check using Proposition 3.4.

As a final step, we need to investigate the term

$$
m(a, b, c)=\mathcal{C}\left(f_{1}(a) f_{2}(b, c)\right)
$$

for all $a, b, c \in \mathfrak{C}$. Since $f_{2}(b, c)$ is 8-periodic, we have

$$
m\left(a s^{2 h}, b s^{i}, c s^{j}\right)=m(a, b, c) s^{2 h+i+j}
$$

for all integers $h, i, j$ and $a, b, c \in \mathfrak{C}$. Therefore, it is enough to consider all triples $(a, b, c) \in(\mathcal{B} \cup \mathcal{B} s) \times \mathcal{B} \times \mathcal{B}$.

Consider the case $a \in \mathcal{B}$. If $a=1$, then $\mathcal{C}\left(f_{1}(a) f_{2}(b, c)\right)=\mathcal{C}\left(f_{2}(b, c)\right)=0$. If $a \in$ $\left\{y^{2}, x^{2} y\right\}$, then $f_{1}(a) f_{2}(b, c)$ is a sum of terms $\beta \bar{p} \alpha, \beta \bar{r} \alpha, \beta \bar{w} \alpha$, and $\beta \bar{x} \bar{y} \alpha$, where $\alpha$ and $\beta$ are monomials in $\bar{x}$ and $\bar{y}$, the degree of $\beta$ is at least 2 , and $\beta \neq \bar{x}^{2}$. Hence, $\mathcal{C}\left(f_{1}(a) f_{2}(b, c)\right)=0$ by Proposition 3.4.

Next, consider $a=x$. By Proposition 3.4 we get $\mathcal{C}\left(\bar{x} f_{2}(b, c)\right)$ from $f_{2}(b, c)$ by the following rule: Put an $\bar{x}$ in front of all monomials in $\bar{x}$ and $\bar{y}$. Then remove all summands containing $\bar{p}, \bar{r}$, or $\bar{w}$, except those beginning with $\bar{p}, \bar{x} \bar{p}$, or $\bar{y} \bar{p}$, where we replace the $\bar{p}$ by $x y$, and $\bar{x} \bar{p}$ and $\bar{y} \bar{p}$ by $x^{2} y$. Finally, replace all $\bar{x}$ and $\bar{y}$ by $x$ and $y$, respectively. Using this procedure, we get the following table for $\mathcal{C}\left(\bar{x} f_{2}(b, c)\right)$ : 


\begin{tabular}{|c|c|c|c|c|c|c|}
\hline $\mathcal{C}\left(\bar{x} f_{2}(b, c)\right)$ & 1 & $\gamma$ & $c$ & $x^{2}$ & $u^{2}$ & $x^{2}$ \\
\hline 1 & 0 & 0 & 0 & 0 & 0 & 0 \\
\hline$x$ & 0 & 0 & 0 & 0 & 0 & 0 \\
\hline$y$ & 0 & $x y$ & 0 & $x y x+x^{2} y+x^{2} y$ & 0 & $*$ \\
\hline$x^{2}$ & 0 & 0 & 0 & $*$ & $*$ & $*$ \\
\hline$y^{2}$ & 0 & $x^{2} y+x y x+x^{2} y+x^{2} y$ & 0 & * & $*$ & $*$ \\
\hline$x^{2} y$ & 0 & $*$ & $*$ & $*$ & $*$ & $*$ \\
\hline
\end{tabular}

Here each $*$ stands for some homogeneous polynomial in $x, y$ of degree at least 4 . Almost all these expressions vanish, and the only remaining terms are

$$
\begin{aligned}
m(x, y, x) & =x y, \\
m\left(x, y, x^{2}\right) & =x^{2} y .
\end{aligned}
$$

For the case $a=y$ we use a similar method resulting from Proposition 3.4, and we end up with $m(y, b, c)=0$ for all $b, c \in \mathcal{B}$. Finally, for $a=x^{2}$ we find that the only non-zero term is $m\left(x^{2}, y, x\right)=x^{2} y$.

The case $a \in \mathcal{B} s$ is slightly more difficult. Consider the map

$$
h(b, c)=\bar{s} f_{2}(b, c) \bar{s}^{-1}-f_{2}(b, c),
$$

measuring how far away $f_{2}$ is from 4 -periodicity. From the equations

\begin{tabular}{|c|c|c|c|c|c|c|c|}
\hline \multicolumn{2}{|c|}{$h(b, c)$} & \multicolumn{4}{|c|}{$c$} & $y^{2}$ & $x^{2} y$ \\
\hline \multirow{6}{*}{$b$} & 1 & 0 & 0 & 0 & 0 & 0 & 0 \\
\hline & $x$ & 0 & 0 & $\bar{x}+\bar{y}$ & $\bar{x}^{2}$ & $\bar{x} \bar{y}$ & $\bar{x}^{2} \bar{y}$ \\
\hline & $y$ & 0 & $\bar{x}+\bar{y}$ & 0 & 0 & $\bar{y}^{2}$ & $\bar{y} \bar{x} \bar{y}+\bar{x} \bar{y}^{2}$ \\
\hline & $x^{2}$ & 0 & $\bar{x}^{2}$ & 0 & $\bar{x}^{3}$ & 0 & $*$ \\
\hline & $y^{2}$ & 0 & $\bar{y} \bar{x}$ & $\bar{y}^{2}$ & 0 & $\bar{y}^{3}$ & * \\
\hline & $x^{2} y$ & 0 & $\bar{x}^{2} \bar{y}$ & 0 & $*$ & $*$ & $*$ \\
\hline
\end{tabular}

$$
\begin{aligned}
\bar{s} \bar{p} \bar{s}^{-1} & =\bar{p}, \\
\bar{s} \bar{r} \bar{s}^{-1} & =\bar{r}+\bar{x}+\bar{y}, \\
\bar{s} \bar{w} \bar{s}^{-1} & =\bar{w}+\bar{y}^{2},
\end{aligned}
$$

\begin{tabular}{|c|c|c|c|c|c|c|c|}
\hline \multirow{2}{*}{\multicolumn{2}{|c|}{$\mathcal{C}(h(b, c))$}} & \multicolumn{6}{|r|}{2} \\
\hline & & 1 & $x$ & $y$ & $x^{2}$ & $y^{2}$ & $x^{2} y$ \\
\hline \multirow{6}{*}{$b$} & 1 & 0 & 0 & 0 & 0 & 0 & 0 \\
\hline & $x$ & 0 & 0 & $x+y$ & $x^{2}$ & $x^{2}+y^{2}$ & $x^{2} y$ \\
\hline & $y$ & 0 & $x+y$ & 0 & 0 & $y^{2}$ & 0 \\
\hline & $x^{2}$ & 0 & $x^{2}$ & 0 & 0 & 0 & 0 \\
\hline & $y^{2}$ & 0 & $x^{2}+y^{2}$ & $y^{2}$ & 0 & 0 & 0 \\
\hline & $x^{2} y$ & 0 & $x^{2} y$ & 0 & 0 & 0 & 0 \\
\hline
\end{tabular}

we get the following table for $h$ :

where $*$ denotes certain homogeneous polynomials in $\bar{x}$ and $\bar{y}$ of degree at least 4 . Applying $\mathcal{C}$ to this table and using relations in $\hat{H}^{*}(G)$, we get 
By definition of $h$, we have $h(b, c) \bar{s}=\bar{s} f_{2}(b, c)-f_{2}(b, c) \bar{s}$; hence

$$
\mathcal{C}(h(b, c)) s=\mathcal{C}\left(\bar{s} f_{2}(b, c)\right)-\underbrace{\mathcal{C}\left(f_{2}(b, c)\right)}_{0} s=m(s, b, c) .
$$

Therefore, this table shows the values $m(s, b, c)$ with $b, c \in \mathcal{B}$. On the other hand, we know that $m$ is a Hochschild-cocycle; in particular, for all $a, b, c \in \mathcal{B}$,

$$
a m(s, b, c)+m(a s, b, c)+m(a, s b, c)+m(a, s, b c)+m(a, s, b) c=0 .
$$

Using $m(a, s, b) c=m(a, 1, b) s c=0, m(a, s, b c)=m(a, 1, b c) s=0$, and $m(a, s b, c)=$ $m(a, b, c) s$, we get

$$
m(a s, b, c)=a m(s, b, c)+m(a, b, c) s .
$$

We know the right-hand side for all $a, b, c \in \mathcal{B}$. Gathering all results, we get the following theorem.

Theorem 3.6. The canonical element $\gamma_{G}$ is represented by the Hochschild cocycle $m$ which is given by the formulae

$$
\begin{aligned}
m(x, y, x) & =x y, & & \\
m\left(x, y, x^{2}\right) & =x^{2} y & & \\
m\left(x^{2}, y, x\right) & =x^{2} y, & & \text { for all other } a, b, c \in \mathcal{B}, \\
m(a, b, c) & =0 & & \\
m(s a, b, c) & =\sin (a, b, c)+s a \mathcal{C}(h(b, c)), & & \text { where } \mathcal{C}(h(b, c)) \text { is given by }(3), \\
m\left(s^{2 i} a, s^{j} b, s^{l} c\right) & =s^{2 i+j+l} m(a, b, c) . & &
\end{aligned}
$$

The element $\gamma \in H H^{3,-1} \hat{H}^{*}(G)$ represented by $m$ is non-trivial.

Proof. It remains to prove the non-triviality of $\gamma$. Assume $m=\delta g$ for some Hochschild $(2,-1)$-cochain $g$. Then,

$$
m(a, b, c)=(\delta g)(a, b, c)=a g(b, c)+g(a b, c)+g(a, b c)+g(a, b) c
$$

for all $a, b, c$. In particular,

$$
\begin{aligned}
0 & =m(y, x, y)=y g(x, y)+g(y x, y)+g(y, x y)+g(y, x) y, \\
0 & =m(x, y, y)=x g(y, y)+g(x y, y)+g\left(x, y^{2}\right)+g(x, y) y, \\
0 & =m(y, y, x)=y g(y, x)+g\left(y^{2}, x\right)+g(y, y x)+g(y, y) x, \\
0 & =m(x, x, x)=x g(x, x)+g\left(x^{2}, x\right)+g\left(x, x^{2}\right)+g(x, x) x, \\
x y & =m(x, y, x)=x g(y, x)+g(x y, x)+g(x, y x)+g(x, y) x .
\end{aligned}
$$

Adding up these equations, we get (using $x^{2}+y^{2}=x y$ )

$$
x y=x \cdot(g(x, y)+g(y, x)) .
$$

This implies $g(x, y)+g(y, x)=y$. On the other hand, interchanging the roles of $x$ and $y$ we get $g(x, y)+g(y, x)=x$, a contradiction. 


\subsection{Computation for the generalized quaternion group}

From now on, we assume that $t \geqslant 4$. Then there is an 8-periodic null-homotopy $\bar{v}$ for $\bar{x}^{2}+\bar{x} \bar{y}$, partially given by

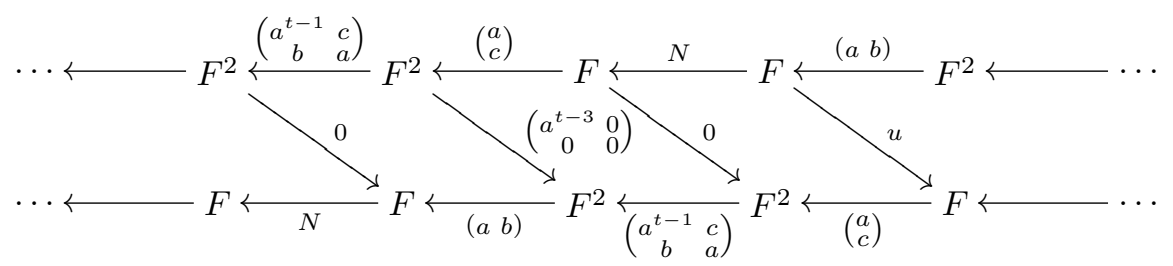

satisfying $\bar{s} \bar{v}+\bar{v} \bar{s}=\bar{x}$. Here we write $u=c a^{2 t-2}+b a^{2 t-3}$ and need to prove

$$
\begin{aligned}
a u & =a^{2 t-2} b+a^{2 t-1}, & c u & =a^{2 t-2} b+a^{2 t-1}, \\
u a & =a^{2 t-2} b+N, & u b & =a^{2 t-2} b .
\end{aligned}
$$

For instance, to prove the first formula, note that

$$
a u+a c a^{2 t-2}=a b a^{2 t-3}=a^{2 t-3} b a=a^{2 t-3} a c=c a^{2 t-2}=(a+b+a c) a^{2 t-2} .
$$

The other formulae can be proved similarly.

Again one verifies that $x^{2} y \neq 0$, so that we recover the well-known structure of $\hat{H}^{*}(G)$ to be

$$
\hat{H}^{*}(G) \cong k\left[x, y, s^{ \pm 1}\right] /\left(y^{3}, x^{2}+x y\right) .
$$

Using the variable $z=x+y$, we obtain the isomorphism

$$
\hat{H}^{*}(G) \cong k\left[x, z, s^{ \pm 1}\right] /\left(x z, x^{3}+z^{3}\right) .
$$

In the following, we will frequently switch between these two descriptions.

Proposition 3.7. We have the following formulae:

$$
\begin{aligned}
\mathcal{C}(\bar{p} \alpha) & =0, & \mathcal{C}(\bar{v} \alpha)=0, & \mathcal{C}(\bar{w} \alpha)=0, \\
\mathcal{C}(\bar{x} \bar{p} \alpha) & =x^{2} \mathcal{C}(\alpha), & \mathcal{C}(\gamma \bar{v} \alpha)=0, & \mathcal{C}(\gamma \bar{w} \alpha)=0, \\
\mathcal{C}(\bar{y} \bar{p} \alpha) & =0, & & \\
\mathcal{C}\left(\bar{x}^{2} \bar{p} \alpha\right) & =x^{2} y \mathcal{C}(\alpha), & & \\
\mathcal{C}\left(\bar{y}^{2} \bar{p} \alpha\right) & =0, & & \\
\mathcal{C}(\beta \bar{p} \alpha) & =0, & &
\end{aligned}
$$

for any $\alpha, \beta, \gamma$ monomials in $\bar{x}, \bar{y}$ with $|\beta| \geqslant 3$.

We omit the straightforward proof and turn to the definition of the maps $f_{1}$ and $f_{2}$. As before, let $\mathcal{B}=\left\{1, x, y, x^{2}, y^{2}, x^{2} y\right\}$; we define $f_{1}$ as

$$
f_{1}\left(s^{i} x^{a} y^{b}\right)=\bar{s}^{i} \bar{x}^{a} \bar{y}^{b}
$$

for all $a, b, i \in \mathbb{Z}$ for which $x^{a} y^{b}$ lies in $\mathcal{B}$. Now we define $f_{2}$ on $\mathcal{B} \times \mathcal{B}$ as follows: 


\begin{tabular}{c|ccc|}
$f_{2}(b, c)$ & \multicolumn{4}{c}{$c$} & \\
\hline 1 & 0 & $x$ & 0 \\
\hline$x$ & 0 & 0 & $\bar{v}$ \\
$b$ & 0 & $\bar{p}+\bar{v}$ & 0 \\
$b$ & $\bar{x} \bar{v}$ & 0 \\
$x^{2}$ & 0 & $\bar{y} \bar{p}+\bar{p} \bar{y}+\bar{v} \bar{y}$ & $\bar{w}$ \\
$y^{2}$ & 0 & $\bar{x}^{2} \bar{p}+\bar{x} \bar{v} \bar{y}+\bar{v} \bar{y}^{2}+\bar{x} \bar{w}+\bar{x}^{2} \bar{y}$ & $\bar{v} \bar{y}^{2}+\bar{x} \bar{w}$ \\
$x^{2} y$ & 0 &
\end{tabular}

\begin{tabular}{c|ccc|} 
& \multicolumn{3}{|c}{$c$} \\
& $x^{2}$ & $y^{2}$ & $x^{2} y$ \\
\hline 1 & 0 & 0 & 0 \\
$x$ & $\bar{x} \bar{v}$ & $\bar{v} \bar{y}$ & $\bar{x} \bar{v} \bar{y}+\bar{v} \bar{y}^{2}+\bar{x} \bar{w}$ \\
$b$ & $\bar{p} \bar{x}+\bar{x} \bar{p}+\bar{x}^{2}$ & $\bar{w}$ & $\bar{y} \bar{v} \bar{y}+\bar{p} \bar{y}^{2}+\bar{x} \bar{w}$ \\
$b^{2}$ & $\bar{x}^{2} \bar{v}+\bar{x} \bar{v} \bar{y}+\bar{y} \bar{y}^{2}+\bar{x} \bar{w}$ & $\bar{v} \bar{y}^{2}+\bar{x} \bar{w}$ & $\bar{x}^{2} \bar{v} \bar{y}+\bar{x} \bar{v} \bar{y}^{2}+\bar{x}^{2} \bar{w}$ \\
$y^{2}$ & $\bar{y}^{2} \bar{v}+\bar{y}^{2} \bar{p}+\bar{w} \bar{x}$ & $\bar{w} \bar{y}$ & $\bar{y}^{2} \bar{v} \bar{y}+\bar{y}^{2} \bar{p} \bar{y}+\bar{w} \bar{x} \bar{y}$ \\
$x^{2} y$ & $\bar{x}^{2} \bar{p} \bar{x}+\bar{x} \bar{v} \bar{y} \bar{x}+\bar{v} \bar{y}^{2} \bar{x}+\bar{x} \bar{w} \bar{x}$ & $\bar{x}^{2} \bar{w}$ & $\bar{x}^{2} \bar{y} \bar{v} \bar{y}+\bar{x}^{2} \bar{p} \bar{y}^{2}+\bar{x}^{3} \bar{w}$ \\
\hline
\end{tabular}

Also put $f_{2}\left(s^{i} a, s^{j} b\right)=f_{2}(a, b) \bar{s}^{i+j}$ for all $i, j \in \mathbb{Z}$ and $a, b \in \mathcal{B}$. This function is chosen in such a way that $\mathcal{C}\left(f_{2}(a, b)\right)=0$ for all $a, b \in \mathcal{B}$. One verifies that

$$
\begin{aligned}
m(x, y, x) & =x^{2}, \\
m\left(x^{2}, y, x\right) & =x^{2} y, \\
m\left(x, y, x^{2}\right) & =x^{2} y,
\end{aligned}
$$

and $m$ vanishes on all other triples $(a, b, c) \in \mathcal{B}^{\times 3}$. Let us define $m^{\prime}$ as follows:

$$
m^{\prime}\left(s^{i} a, s^{j} b, s^{k} c\right)=s^{i+j+k} m(a, b, c) \quad \text { for all } a, b, c \in \mathcal{B},
$$

and define $h(a, b)=\bar{s} f_{2}(a, b) \bar{s}^{-1}-f_{2}(a, b)$. Then $\mathcal{C}(h(b, c))$ is given by the following table:

\begin{tabular}{c|cccccc|}
$\mathcal{C}(h(b, c))$ & 1 & $x$ & $y$ & $x^{2}$ & $y^{2}$ & $x^{2} y$ \\
\hline 1 & 0 & 0 & 0 & 0 & 0 & 0 \\
& 0 & 0 & $x$ & $x^{2}$ & $x^{2}$ & $x^{2} y$ \\
$b$ & 0 & $x$ & 0 & 0 & $y^{2}$ & 0 \\
$b$ & 0 & $x^{2}$ & 0 & 0 & 0 & 0 \\
$x^{2}$ & 0 & $x^{2}$ & $y^{2}$ & 0 & 0 & 0 \\
$y^{2}$ & 0 & $x^{2} y$ & 0 & 0 & 0 & 0 \\
$x^{2} y$ & 0
\end{tabular}

So we get the following explicit description of $m$ :

Theorem 3.8. The canonical element $\gamma_{G}$ is represented by the Hochschild cocycle $m$ 
which is given by the formulae:

$$
\begin{array}{rlrl}
m(x, y, x) & =x^{2}, & & \\
m\left(x^{2}, y, x\right) & =x^{2} y & & \\
m\left(x, y, x^{2}\right) & =x^{2} y, & & \text { for all other } a, b, c \in \mathcal{B}, \\
m(a, b, c) & =0 & & \\
m(s a, b, c) & =s m(a, b, c)+s a \mathcal{C}(h(b, c)), & \text { where } \mathcal{C}(h(b, c)) \text { is given by }(6), \\
m\left(s^{2 i} a, s^{j} b, s^{l} c\right) & =s^{2 i+j+l} m(a, b, c) . &
\end{array}
$$

The element $\gamma \in H H^{3,-1} \hat{H}^{*}(G)$ represented by $m$ is non-trivial.

Proof. It remains to prove the non-triviality of $\gamma$. Suppose that $m$ is a Hochschild coboundary; then $m=\delta g$ for some $g: \Lambda^{\otimes 2} \rightarrow \Lambda[-1]$. Adding up the equations

$$
\begin{aligned}
x^{3} & =m\left(x, z, x^{2}\right)=x g\left(z, x^{2}\right)+g(x, z) x^{2} \\
0 & =m\left(x^{2}, x, z\right)=x^{2} g(x, z)+g\left(x^{3}, z\right)+g\left(x^{2}, x\right) z \\
0 & =m\left(z, x^{2}, x\right)=z g\left(x^{2}, x\right)+g\left(z, x^{3}\right)+g\left(z, x^{2}\right) x \\
0 & =m\left(z, z^{2}, z\right)=z g\left(z^{2}, z\right)+g\left(z^{3}, z\right)+g\left(z, z^{3}\right)+g\left(z, z^{2}\right) z \\
0 & =z m(z, z, z)=z^{2} g(z, z)+z g\left(z^{2}, z\right)+z g\left(z, z^{2}\right)+z g(z, z) z
\end{aligned}
$$

and simplifying, we get the contradiction $x^{3}=0$.

\section{Realizability of modules}

\subsection{Massey products}

There is a strong connection between the canonical class $\gamma$ and triple Massey products over $\hat{H}^{*}(G)$. This has already been noted in [2, Lemma 5.14], and we will generalize this fact to Massey products of matrices (as introduced by May [5]). We start with some notation. Let $\Lambda$ be a graded $k$-algebra, and suppose that $I$ is a graded set; i.e., a set together with a function $|\cdot|: I \rightarrow \mathbb{Z}$. For every such set, we define $I[n]$ to be the shifted graded set given by the same set with new grading $|i|_{[n]}=|i|+n$ for all $i \in I$. We denote by $\Lambda^{I}$ the shifted free $\Lambda$-module

$$
\Lambda^{I}=\bigoplus_{i \in I} \Lambda[|i|]
$$

Then $\Lambda^{I}[n]=\Lambda^{I[n]}$. If $J$ is another graded set, we can consider morphisms $f: \Lambda^{J} \rightarrow$ $\Lambda^{I}$. Every such map can be represented by a (possibly infinite) matrix $\left(f_{i, j}\right)_{i \in I, j \in J}$ with $\left|f_{i, j}\right|=|i|-|j|$. Such a matrix is column-finite; i.e., for every $j$ there are only finitely many non-zero $f_{i, j}$ 's. Let us denote by $\Lambda^{I, J}$ the set of such matrices. Every such yields a map $f: \Lambda^{J} \rightarrow \Lambda^{I}$.

A triple of matrices $(A, B, C)$ will be called composable if there are graded sets $I, J, K, L$ with $A \in \Lambda^{I, J}, B \in \Lambda^{J, K}, C \in \Lambda^{K, L}$. Every morphism $m: \Lambda^{\otimes 3} \rightarrow \Lambda[-1]$ can be extended to the module of all composable triples by putting

$$
m(A, B, C) \in \Lambda^{I[-1], L}: \quad m(A, B, C)_{i[-1], l}=\sum_{j \in J} \sum_{k \in K} m\left(a_{i j}, b_{j k}, c_{k l}\right) .
$$


From now on we assume $\Lambda=H^{*} \mathcal{A} \cong \hat{H}^{*}(G)$, where $\mathcal{A}$ is the endomorphism-dgA of some projective resolution of the trivial $k G$-module $k$. Also, let $m: \Lambda^{\otimes 3} \rightarrow \Lambda[-1]$ be some Hochschild cocycle representing the canonical element $\gamma \in H H^{3,-1} \hat{H}^{*}(G)$. Recall that (see, e.g., [5]) for every composable triple of matrices $(A, B, C)$ with $A B=0$ and $B C=0$ the triple matric Massey product $\langle A, B, C\rangle$ is defined and a coset of $A \cdot \Lambda^{J[-1], L}+\Lambda^{I[-1], K} \cdot C$. Notice that there is no obstruction to generalizing May's definition to infinite matrices.

Proposition 4.1. For every composable triple $(A, B, C)$ with $A B=0$ and $B C=0$, we have that $m(A, B, C) \in\langle A, B, C\rangle$.

Proof. We have

$$
\begin{aligned}
m(A, B, C) & =f_{1}(A) f_{2}(B, C)+f_{2}(A B, C)+f_{2}(A, B C)+f_{2}(A, B) f_{1}(C) \\
& =f_{1}(A) f_{2}(B, C)+f_{2}(A, B) f_{1}(C),
\end{aligned}
$$

and the last term represents one element of the Massey product.

A triple $(A, B, C)$ will be called exact if it is composable and the sequence

$$
\Lambda^{I} \stackrel{A}{\leftarrow} \Lambda^{J} \stackrel{B}{\longleftarrow} \Lambda^{K} \stackrel{C}{\leftarrow} \Lambda^{L}
$$

is exact.

Proposition 4.2. Let $A \in \Lambda^{I, J}$ be any matrix, and define $M=\operatorname{coker} A$. Then the following are equivalent:

(i) The module $M$ is a direct summand of a realizable module.

(ii) For every composable triple $(A, B, C)$ with $A B=0$ and $B C=0$, we have that $0 \in\langle A, B, C\rangle$.

(iii) For some exact triple $(A, B, C)$, we have $0 \in\langle A, B, C\rangle$.

Proof. For (i) $\Rightarrow$ (ii), let $M$ be a direct summand of $H^{*} N$, where $N$ is some dg- $\mathcal{A}$ module. Then there are maps $M \stackrel{i}{\rightarrow} H^{*} N \stackrel{r}{\rightarrow} M$ with $r i=\operatorname{id}_{M}$. Let $\pi: \Lambda^{I} \rightarrow M$ be the projection map, and put $W=i \pi$. Then $W A=0$, so that $\langle W, A, B\rangle$ is defined, and the juggling formula (see [5, Corollary 3.2.(iii)]) yields $W\langle A, B, C\rangle=\langle W, A, B\rangle C$ as cosets of $W \Lambda^{I-1], K} C$. Let $E: \Lambda^{K} \rightarrow H^{*} N[-1]$ be some element in $\langle W, A, B\rangle$. Since $\Lambda^{K}$ is free, we know that the composition $r \circ E$ lifts as $\Lambda^{K} \stackrel{S}{\rightarrow} \Lambda^{I[-1]} \stackrel{\pi}{\rightarrow} M[-1]$ for some matrix $S$. But then

$$
\pi S C=r E C \in r\langle W, A, B\rangle C=r W\langle A, B, C\rangle=\pi\langle A, B, C\rangle .
$$

This means that there is some matrix $T$ such that $A T+S C \in\langle A, B, C\rangle$, which implies $0 \in\langle A, B, C\rangle$.

The implication (ii) $\Rightarrow$ (iii) is obvious. For (iii) $\Rightarrow$ (i), note that

$$
M \leftarrow \Lambda^{I} \stackrel{A}{\leftarrow} \Lambda^{J} \stackrel{B}{\leftarrow} \Lambda^{K} \stackrel{C}{\leftarrow} \Lambda^{L}
$$

is the beginning of a (shifted) free resolution of $M$. We have $m(A, B, C) \in \Lambda^{I[-1], L}$, and a representative of $\gamma \cup \operatorname{id}_{M} \in \widehat{\operatorname{Ext}}_{\Lambda}^{3,-1}(M, M)$ is given by the composition

$$
g: \Lambda^{L} \stackrel{m(A, B, C)}{\longrightarrow} \Lambda^{I[-1]} \rightarrow(\operatorname{coker} A)[-1]=M[-1] .
$$

By assumption and Proposition 4.1, $m(A, B, C)=A X+Y C$ for some matrices $X$ 
and $Y$, so that this composition equals

$$
\Lambda^{L} \stackrel{C}{\rightarrow} \Lambda^{K} \stackrel{Y}{\rightarrow} \Lambda^{I[-1]} \rightarrow M[-1],
$$

which in turn says that $g$ is the coboundary of $\Lambda^{K} \stackrel{Y}{\rightarrow} \Lambda^{I[-1]} \rightarrow M[-1]$; hence $\gamma \cup$ $\operatorname{id}_{M}=0$. By Theorem 1.1 of [2], $M$ is a direct summand of some realizable module.

\subsection{The group of quaternions}

Let $G=Q_{8}$. We shall make use of one of the implications of Proposition 4.2 to prove the existence of a $\hat{H}^{*} G$-module which detects the non-triviality of $\gamma_{G}$ :

Theorem 4.3. The cokernel of the map

$$
\Lambda[-1] \oplus \Lambda[-1] \stackrel{\left(\begin{array}{cc}
y & x+y \\
x & y
\end{array}\right)}{\longrightarrow} \Lambda \oplus \Lambda
$$

is not a direct summand of a realizable $\hat{H}^{*} G$-module.

Proof. Let $A=\left(\begin{array}{c}y \\ x \\ x+y\end{array}\right)$; then $A^{2}=0$ and therefore the Massey product $\langle A, A, A\rangle$ is defined. We claim that it does not contain 0. An explicit calculation using the description of $m$ given in Theorem 3.6 yields

$$
m(A, A, A)=\left(\begin{array}{cc}
x^{2} & 0 \\
x^{2} & x^{2}
\end{array}\right) .
$$

Let us denote the latter matrix by $B$; then by Proposition 4.2 we need to prove that $B$ is not of the form $B=A Q+R A$ for some $2 \times 2$-matrices $Q$ and $R$. To do so, define $D=\left(\begin{array}{cc}x & y \\ x+y & x\end{array}\right)$; then $A D=D A=0$. If we denote by tr the trace of a matrix, then we have

$$
\operatorname{tr}(B D)=\operatorname{tr}(A Q D)+\operatorname{tr}(R A D)=\operatorname{tr}(Q D A)+\operatorname{tr}(R A D)=0
$$

(note that these computations take place in a commutative ring). But

$$
\operatorname{tr}(B D)=\operatorname{tr}\left(\begin{array}{cc}
0 & * \\
* & x^{2} y
\end{array}\right)=x^{2} y \neq 0
$$

a contradiction.

Remark 4.4. The triple $(A, A, A)$ is actually exact, but we do not need this.

In order to construct a module which is not a direct summand of a realizable one, it is often enough to consider "ordinary" Massey products, i.e., the case of $1 \times 1$ matrices; this is true for example in the cases $G=\mathbb{Z} / 2 \mathbb{Z} \times \mathbb{Z} / 2 \mathbb{Z}$ ([2, Example 7.7]) and $G=\mathbb{Z} / 3 \mathbb{Z}$ (characteristic 3, [2, Example 7.6]). In our present case, it is not that easy:

Proposition 4.5. Let $k=\mathbb{F}_{2}$ be the field with 2 elements. For all $a, b, c \in \hat{H}^{*}\left(Q_{8}\right)$ satisfying $a b=0$ and $b c=0$, we have $0 \in\langle a, b, c\rangle$. 
Proof. By [2, Lemma 5.14], the class $m(a, b, c)$ is contained in the Massey product $\langle a, b, c\rangle$. Therefore, it is enough to show that $m(a, b, c)$ is an element of the indeterminacy

$$
a \cdot \hat{H}^{|b|+|c|-1}(G)+\hat{H}^{|a|+|b|-1}(G) \cdot c
$$

for all $a, b, c$. By construction of $m$ it is enough to do so for those triples $(a, b, c)$ and $(s a, b, c)$ with $a, b, c \in\left\{1, x, y, x+y, x^{2}, y^{2}, x^{2}+y^{2}, x^{2} y\right\}$ which satisfy $a b=0$ and $b c=0$.

If $|a|,|b| \leqslant 1$, then $a b=0$ implies $a=0$ or $b=0$ (here we use that $k=\mathbb{F}_{2}$ ). If $|b| \geqslant 2$, then $m(a, b, c)=0$ unless $b \in\left\{y^{2}, y^{2}+x^{2}\right\}$ and $a, c \in\{x, x+y\}$, in which case $m(a, b, c)=x^{2} y$ is divisible by $a$. So we can assume that $|b|=1$ and therefore $|a| \geqslant 2$ and $|c| \geqslant 2$, which implies $m(a, b, c)=0$ by Theorem 3.6.

For $m(s a, b, c)$, we have by $(4)$

$$
m(s a, b, c)=a m(s, b, c)+m(a, b, c) s .
$$

We have already seen that the second summand lies in the indeterminacy; the first summand is contained in

$$
a \cdot \hat{H}^{|s|+|b|+|c|-1}(G)=s a \cdot \hat{H}^{|b|+|c|-1}(G)
$$

and therefore in the indeterminacy.

Remark 4.6. Note that the proposition is not true for arbitrary fields of characteristic 2: If the field $k$ contains an element $\alpha \in k$ satisfying $\alpha^{2}+\alpha+1=0$, then the Massey product

$$
\left\langle\alpha x+y, \alpha^{2} x+y, \alpha x+y\right\rangle
$$

is defined and does not contain 0 .

\subsection{Generalized quaternions}

The picture changes as soon as we consider generalized quaternion groups $G=Q_{4 t}$ with $t \geqslant 4$. It turns out that there is no module detecting the non-triviality of the canonical element $\gamma_{G}$.

Let $m$ be as in Theorem 3.8, and write $m=m^{\prime}+m^{\prime \prime}$, where $m^{\prime}$ is defined in (5). Notice that $m^{\prime}$ is a Hochschild cocycle, because it is defined to be $s$-periodic, so it is enough to check the cocycle condition on elements in $\mathcal{B}$. But on these elements, $m^{\prime}$ agrees with $m$. Hence, $m^{\prime}$ is a cocycle, and so is $m^{\prime \prime}$. Let $\gamma^{\prime}$ and $\gamma^{\prime \prime}$ be the corresponding elements in $H H^{3,-1} \hat{H}^{*}(G)$. In the next two propositions we will show that, for every module $M, \gamma^{\prime} \cup \operatorname{id}_{M}=0$ and $\gamma^{\prime \prime} \cup \operatorname{id}_{M}=0$ in $\operatorname{Ext}^{3,-1}(M, M)$, respectively. It will then follow that $M$ is a direct summand of a realizable module.

Proposition 4.7. For every $\Lambda$-module $M$ we have $\gamma^{\prime} \cup \mathrm{id}_{M}=0$.

Proof. Notice that every matrix $A \in \Lambda^{I, J}$ can be uniquely written as a sum

$$
A=A_{1}+A_{x} x+A_{y} y+A_{x^{2}} x^{2}+A_{y^{2}} y^{2}+A_{x^{2} y} x^{2} y,
$$

where the six matrices on the right-hand side lie in $k\left[s^{ \pm 1}\right]^{I, J[?]}$. The first step in our proof will be to find a suitable free resolution

$$
M \leftarrow \Lambda^{I} \stackrel{A}{\leftarrow} \Lambda^{J} \stackrel{B}{\longleftarrow} \Lambda^{K} \stackrel{C}{\longleftarrow} \Lambda^{L}
$$

of $M$. We begin with the definition of $A$. Let $I$ be a minimal set of generators of the 
right $\Lambda$-module $M$; i.e., $I$ generates $M$ but any proper subset of $I$ does not generate $M$ (in the case where $M$ is not finitely generated, one has to use Zorn's lemma to prove the existence of $I$ ). The inclusion $I \subseteq M$ induces a surjection $\Lambda^{I} \rightarrow M$. Let $J$ be a minimal set of generators for the kernel of that map; then we obtain an exact sequence $\Lambda^{J} \stackrel{A}{\rightarrow} \Lambda^{I} \rightarrow M$. Taking $K$ to be a minimal set of generators for the kernel of $A$, we get a map $\Lambda^{K} \stackrel{B}{\rightarrow} \Lambda^{J}$ onto that kernel, and finally we let $L$ be a minimal set of generators for the kernel of $B$ to obtain an exact sequence

$$
M \leftarrow \Lambda^{I} \stackrel{A}{\leftarrow} \Lambda^{J} \stackrel{B}{\longleftarrow} \Lambda^{K} \stackrel{C}{\longleftarrow} \Lambda^{L} .
$$

We claim that $A_{1}=0$. Assume the contrary and let $i \in I, j \in J$ be such that $\left(A_{1}\right)_{i, j} \neq$ 0 . Then $I-\{i\}$ generates $M$, which contradicts the choice of $I$. Similarly one shows that $B_{1}=0$ and $C_{1}=0$, and therefore $B_{y} C_{y}=(B C)_{y^{2}}=0$.

Now define $W=A_{x} B_{y} x+A_{x^{2}} B_{y} x^{2}$ and $V=B_{y} C_{y^{2}} y^{2}$. Then

$$
\begin{aligned}
A V= & A_{x} B_{y} C_{y^{2}} x^{3}, \\
W C= & A_{x} B_{y} C_{x} x^{2}+A_{x} \underbrace{B_{y} C_{y}}_{0} x^{2}+A_{x} B_{y} C_{x^{2}} x^{3}+A_{x} B_{y} C_{y^{2}} x^{3} \\
& +A_{x^{2}} B_{y} C_{x} x^{3}+A_{x^{2}} \underbrace{B_{y} C_{y}}_{0} x^{3} .
\end{aligned}
$$

Therefore, $m^{\prime}(A, B, C)=A V+W C$, and by Proposition 4.2 we get $\gamma^{\prime} \cup \operatorname{id}_{M}=0$.

Proposition 4.8. For every $\Lambda$-module $M$, we have $\gamma^{\prime \prime} \cup \operatorname{id}_{M}=0$.

Proof. We start with a slight modification of the representative $m^{\prime \prime}$. Let us put $\mathcal{B}=$ $\left\{1, x, z, x^{2}, z^{2}, x^{3}\right\}$, and define the function $g$ as follows: For all integers $i$, put

$$
\begin{aligned}
& g\left(s^{-1} x^{2}, s^{i} x\right)=s^{i-1} z^{2}, \\
& g\left(s^{-1} x^{2}, s^{i} z\right)=s^{i-1} x^{2},
\end{aligned}
$$

and $g(a, b)=0$ on all other elements $a, b$ in $\left\{s^{i} c \mid c \in \mathcal{B}\right\}$. Then $\tilde{m}=m^{\prime \prime}+\partial g$ defines a new representative for the element $\gamma^{\prime \prime}$. For all $a, b, c \in \mathcal{B}$ and $i, j \geqslant 1$, we have

$$
\begin{aligned}
\tilde{m}\left(a, s^{i} b, s^{j} c\right)=m^{\prime \prime}\left(a, s^{i} b, s^{j} c\right)+a g\left(s^{i} b, s^{j} c\right)+g\left(s^{i} a b, s^{j} c\right) & \\
& +g\left(a, s^{i+j} b c\right)+g\left(a, s^{i} b\right) s^{j} c
\end{aligned}
$$

and by definition of $m^{\prime \prime}$ and $g$ each summand on the right-hand side vanishes. We also have that

$$
\begin{aligned}
\tilde{m}\left(s^{-1} a, s^{i} b, s^{j} c\right)= & m^{\prime \prime}\left(s^{-1} a, s^{i} b, s^{j} c\right)+s^{-1} a \underbrace{g\left(s^{i} b, s^{j} c\right)}_{0}+\underbrace{g\left(s^{i-1} a b, s^{j} c\right)}_{0} \\
& +g\left(s^{-1} a, s^{i+j} b c\right)+g\left(s^{-1} a, s^{i} b\right) s^{j} c .
\end{aligned}
$$

We claim that this is zero if $|a| \geqslant 2,|b| \geqslant 1$, and $|c| \geqslant 1$. In that case, we have $|b c| \geqslant 2$ and therefore $g\left(s^{-1} a, s^{i+j} b c\right)=0$, so that it remains to show $m^{\prime \prime}\left(s^{-1} a, s^{i} b, s^{j} c\right)=$ 
$g\left(s^{-1} a, s^{i} b\right) s^{j} c$, or equivalently,

$$
m^{\prime \prime}\left(s^{-1} a, b, c\right)=g\left(s^{-1} a, b\right) c .
$$

To see this, we consider the several cases for $a$ separately. If $a=x^{3}$, then

$$
m^{\prime \prime}\left(s^{-1} a, b, c\right)=s^{-1} x^{3} \mathcal{C}(h(b, c)),
$$

where $h$ is as in Theorem 3.8. But $|h(b, c)| \geqslant 1$, so the last expression vanishes, as does $g\left(s^{-1} a, b\right) c$. For $a=z^{2}$ we get

$$
m^{\prime \prime}\left(s^{-1} a, b, c\right)=s^{-1} z^{2} \mathcal{C}(h(b, c)),
$$

but $|h(b, c)| \geqslant 2$ or $\mathcal{C}(h(b, c))$ is divisible by $x$, and therefore again the right-hand side vanishes. The last case is $a=x^{2}$, where we need to show

$$
s^{-1} x^{2} \mathcal{C}(h(b, c))=g\left(s^{-1} x^{2}, b\right) c .
$$

Both sides vanish for degree reasons unless $|b|=|c|=1$, and in that case both sides will equal $s^{-1} x^{3}$ if $b \neq c$, and 0 otherwise.

The rest is easy. We start with a free resolution of $M$ as in the proof of Proposition 4.7. We can (and do) assume that the degree $|i|$ of every element $i \in I$ lies in $\{0,1,2,3\}$. Also, we assume that the degree of every element of $J$ lies in $\{-1,0,1,2\}$, the degree of every element of $K$ belongs to $\{-8,-7,-6,-5\}$, and the degree of every element of $L$ is in $\{-15,-14,-13,-12\}$. Then we know that every non-zero entry of $B$ and $C$ is a linear combination of terms of the form $s^{i} b$ with $i \geqslant 1$ and $b \in \mathcal{B}$, $|b| \geqslant 1$. Furthermore, every non-zero entry of $A$ is a linear combination of elements in $\mathcal{B} \cup\left\{s^{-1} x^{2}, s^{-1} z^{2}, s^{-1} x^{3}\right\}$. By what we have shown above, $\tilde{m}(A, B, C)=0$, and we are done.

\section{References}

[1] A. Adem and R.J. Milgram, Cohomology of finite groups, second edition, Grundlehren der Mathematischen Wissenschaften [Fundamental Principles of Mathematical Sciences], vol. 309, SpringerVerlag, Berlin, 2004.

[2] D. Benson, H. Krause, and S. Schwede, Realizability of modules over Tate cohomology, Trans. Amer. Math. Soc. 356 (2004), no. 9, 3621-3668.

[3] J.F. Carlson, Modules and group algebras, Lectures in Mathematics ETH Zürich, Birkhäuser Verlag, Basel, 1996.

[4] H. Cartan and S. Eilenberg, Homological algebra, Princeton Landmarks in Mathematics, Princeton University Press, Princeton, NJ, 1999.

[5] J.P. May, Matric Massey products, J. Algebra 12 (1969), 533-568.

Martin Langer martinlanger@yahoo.com

Westfälische Wilhelms-Universität Münster, Institut für Mathematik, Einsteinstr. 62, 48149 Münster, Germany 